\title{
Normal Acceleration Reduce Guidance and Control for Sub-orbit Vehicle Re-entry
}

\author{
Wenhao $\mathrm{Li}^{1}$, Xinxin $\mathrm{Xiao}^{2}$ and Heng Zhang ${ }^{3}$ \\ Institute of Mechanics, Chinese Academy of Sciences, Beijing 100190, P. R. China
}

\begin{abstract}
In this paper, a new angle-of-attack(AOA) guidance law and controller with multi-control surface and reaction control system (RCS) are designed to reduce normal acceleration in suborbital re-entry phase. First, the six-degree-of-freedom (6 DOF) nonlinear dynamic model of sub-orbital vehicles established, and the overload characteristics is analyzed under traditional AOA guidance law. Second, an AOA guidance law added height element is proposed to reduce the Normal accelerate peak value during re-entry. Furthermore, an Inverse-PIDA control law and composite control strategy are designed to control multi-aerodynamic surface and RCS. Finally the proposed approach is applied in a sub-orbital vehicle re-entry flight, validation simulation are conducted by using the proposed guidance law and controller with the 6 DOF nonlinear dynamic model , Monte-Carlo statistical analysis shows it can get well control performance with $\pm \mathbf{2 0} \%$ aerodynamic elements uncertainty and random wind.
\end{abstract}

\section{Introduction}

Sub-orbital vehicle is a low operating costs, usually reusable shuttle; it has great potential to applicate in space tourism, rapid long-range deployment and low cost launch vehicle. Compare with the Space shuttle, the initial height of sub-orbital vehicle re-entry is approximately (about 100km), but the Initial velocity is much lesser (the Space shuttle is about $8 \mathrm{~km} / \mathrm{s}$, but sub-orbital vehicle generally lesser than $2 \mathrm{~km} / \mathrm{s}$ ). The low initial velocity will let to a large reentry path angle (For example the" Spaceship One/SpaceShipTwo"1, as show in Fig. 1, so sub-orbital vehicle will rapidly entering dense atmosphere without sufficiently decelerate, that will cause highly pneumatic overload (normal acceleration may be higher than $5 \mathrm{G}$, or even more higher, but the normal acceleration of the Space Shuttle is lesser than 3G in re-entry phase ). Highly normal acceleration treat the safety of passenger, cargo and vehicle's body, and that is an important problem which may restrained the application of sub-orbital vehicle.

Previous studies of sub-orbital re-entry usually use the Space Shuttle's AOA design based on re-entry corridor analyze method. Researchers found that overload peak would be very high during re-entry ${ }^{2-4}$. Liu Quan-jun proposed the upper-limit of altitude for sub-orbital vehicle, because of the dynamic pressure and overload constraints ${ }^{5}$. Xiao xin-xin proposed "re-entry channel" concept ${ }^{6}$ instead of re-entry corridor to analyze sub-orbital re-entry, and she gave a re-entry AOA design method based on normal overload dynamic equilibrium to reduce normal acceleration peak value $^{7}$; Wu Liao-ni divided reusable launch vehicle (RLV) re-entry AOA guidance into three sequential phase: alpha recovery, Nz hold and alpha transition, he use predictor-corrector iterative guidance algorithm ${ }^{8}$ to maintain overload so to reduce dynamic pressure.

In this paper, we aim to reduce the normal acceleration during sub-orbital vehicle re-entry, a new angle-of-attack (AOA) guidance law and controller with multi-control surface and reaction control system (RCS) are designed.

1 Associate Professor, Institute of Mechanics, Chinese Academy of Sciences, liwenhao@imech.ac.cn, and AIAA Member Grade for first author.

${ }_{2}$ Aassistant Professor, Institute of Mechanics, Chinese Academy of Sciences, xiaoxinxin@imech.ac.cn, and AIAA Member Grade for second author.

3 Professor, Institute of Mechanics, Chinese Academy of Sciences, hzhang@imech.ac.cn, and AIAA Member Grade for third author. 


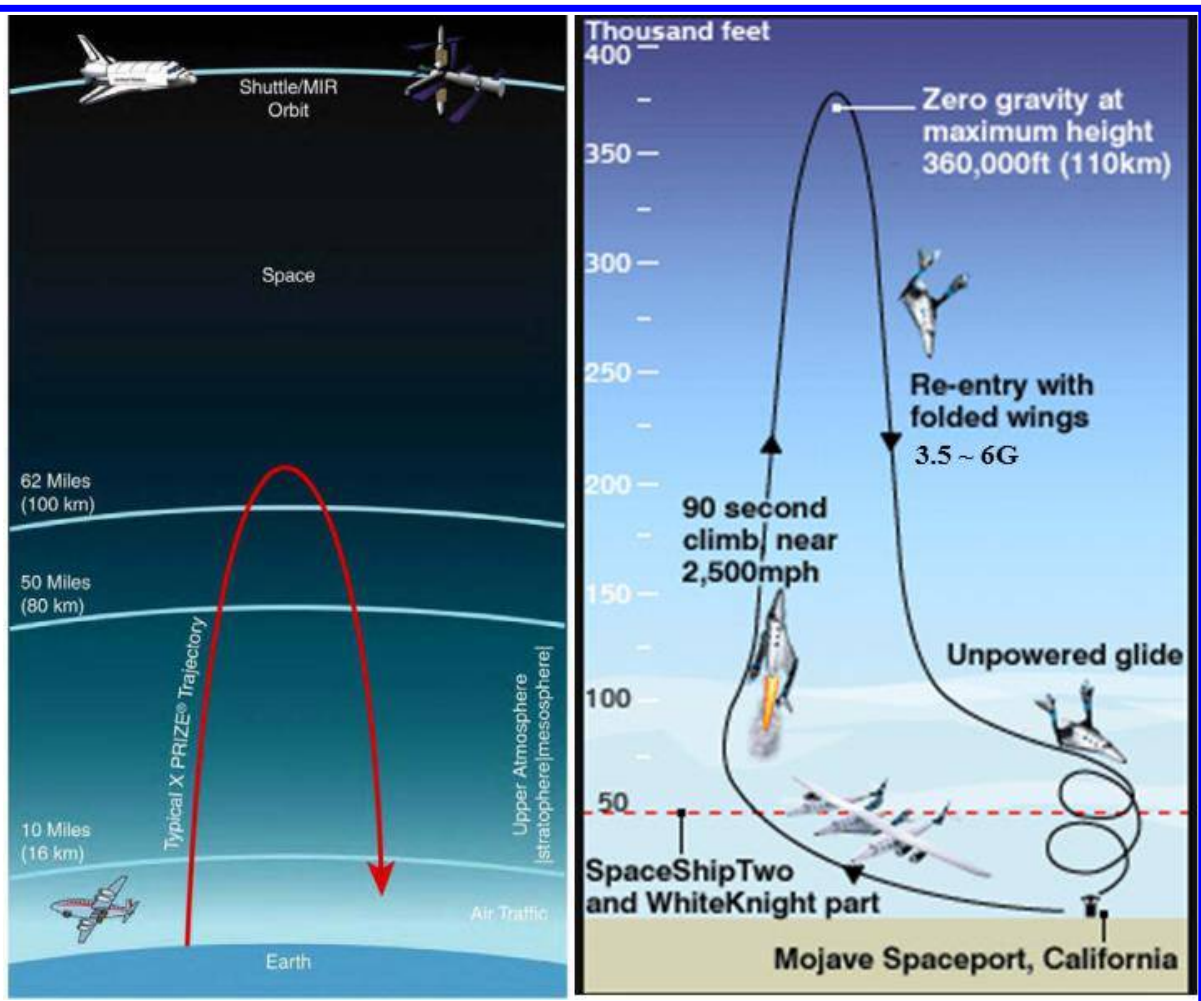

Figure 1. Different re-entry process. Figure show the differences between sub-orbit vehicle and the space shuttle during re-entry (left), diagram of the Spaceship One/Two flight track (right). Spaceship One/Two use folded wings to slow down the re-entry speed, even so, passengers experience force will up to $6 \mathrm{G}$ (about 2 times as the Max overload of Space Shuttle re-entry ).

\section{Re-entry model}

\section{A. 6 DOF dynamics and kinematics model}

The well-known point-mass dynamics of entry vehicle over earth ellipsoid model described by the following equations:

$$
\begin{aligned}
& \frac{d r}{d t}=v \sin \gamma \\
& \frac{d \lambda}{d t}=\frac{v \sin \gamma \cos \xi}{r \cos \psi} \\
& \frac{d \psi}{d t}=\frac{v \cos \gamma \sin \xi}{r} \\
& \frac{d v}{d t}=-\frac{1}{m} D-g_{r} \sin \gamma+\omega^{2} r \cos \psi(\sin \gamma \cos \psi-\cos \gamma \sin \xi \sin \psi)
\end{aligned}
$$

$v \frac{d \gamma}{d t}=\frac{1}{m} L \cos \sigma-g_{r} \cos \gamma+\frac{v^{2}}{r} \cos \gamma+2 \omega v \cos \xi \cos \psi+\omega^{2} r \cos \psi(\cos \gamma \cos \psi+\sin \gamma \sin \xi \sin \psi)$ $v \frac{d \xi}{d t}=-\frac{1}{m} \frac{L \cos \sigma}{\cos \gamma}-\frac{v^{2}}{r} \cos \gamma \cos \xi \tan \psi+2 \omega v(\tan \gamma \sin \xi \cos \psi-\sin \psi)-\frac{\omega^{2} r}{\cos \gamma} \cos \psi \sin \psi \cos \xi$

$$
-g_{\psi} \frac{\sin \xi \cos \xi}{\cos \gamma}
$$

where $\mathrm{r}$ is the radial distance from the center of the earth to the vehicle, the longitude and latitude are $\lambda$ and $\psi$, respectively, $v$ is the earth-relative velocity, the flight path angle is $\gamma$ and $\sigma$ the bank angle, the velocity azimuth angle 
$\psi$ is measured from the north in a clockwise direction, the earth rotation terms are considered in above equation, where $g_{r}$ and $g_{\psi}$ are longitudinal and latitudinal direction of the gravity acceleration, and $\omega$ is the earth rotation velocity.

The Terms $D$ and $L$ are the aerodynamic drag and lift, where $\rho$ is the atmospheric density, $S$ is the reference area of the vehicle, and $m$ is the mass. Note that $D$ and $L$ are also functions of the angle of attack $\alpha$, through the dependence of the drag and lift coefficients $C_{D}$ and $C_{L}$ on $\alpha$.

The overload constraints during re-entry is

$$
\begin{aligned}
D & =\frac{1}{2} \rho v^{2} S C_{D} \\
L & =\frac{1}{2} \rho v^{2} S C_{L}
\end{aligned}
$$

$$
\begin{aligned}
& N_{x}=\frac{F_{x}}{G} \leq N_{x \_ \text {max }} \\
& N_{n}=\frac{F_{n}}{G} \leq N_{n \_ \text {max }}
\end{aligned}
$$

where $F_{x}, F_{n}$ is the longitudinal and normal force on the aircraft, that $F_{x}=L \sin \alpha-D \cos \alpha$ and $F_{n}=-L \cos \alpha-$ $D \sin \alpha$.

In order to discuss the attitude change during the re-entry, the attitude dynamics and kinematics equations are established as follows

$$
\begin{gathered}
I_{x} \frac{d \omega}{d t}=\left(I_{y}-I_{z}\right) \omega_{z} \omega_{y}-I_{y z}\left(\omega_{y}^{2}-\omega_{z}^{2}\right)-I_{z x}\left(\frac{d \omega_{z}}{d t}+\omega_{x} \omega_{y}\right)-I_{x y}\left(\frac{d \omega_{y}}{d t}-\omega_{z} \omega_{x}\right)-M_{x} \\
I_{y} \frac{d \omega}{d t}=\left(I_{z}-I_{x}\right) \omega_{x} \omega_{z}-I_{z x}\left(\omega_{z}^{2}-\omega_{x}^{2}\right)-I_{x y}\left(\frac{d \omega_{z}}{d t}+\omega_{y} \omega_{z}\right)-I_{y z}\left(\frac{d \omega_{z}}{d t}-\omega_{y} \omega_{x}\right)-M_{y} \\
I_{z} \frac{d \omega}{d t}=\left(I_{x}-I_{y}\right) \omega_{x} \omega_{y}-I_{x y}\left(\omega_{x}^{2}-\omega_{y}^{2}\right)-I_{y z}\left(\frac{d \omega_{z}}{d t}+\omega_{z} \omega_{x}\right)-I_{z x}\left(\frac{d \omega_{x}}{d t}-\omega_{y} \omega_{z}\right)-M_{z} \\
\omega_{x}=\dot{\varphi}+\sin \theta \dot{\phi} \\
\omega_{y}=\sin \varphi \dot{\theta}+\cos \varphi \cos \theta \dot{\varphi} \\
\omega_{z}=\cos \varphi \dot{\theta}-\sin \varphi \cos \theta \dot{\varphi}
\end{gathered}
$$

The attitude control model of the re-entry vehicle can described in the following formula

$$
\begin{gathered}
I \dot{\boldsymbol{\omega}}=-\boldsymbol{\Omega I} \boldsymbol{\omega}+\boldsymbol{M} \\
\dot{\boldsymbol{\gamma}}=R(\phi) \boldsymbol{\omega} \\
\boldsymbol{y}=\boldsymbol{\gamma}
\end{gathered}
$$

where $I$ Is Inertia tensor, $\boldsymbol{\omega}=\left[\omega_{x}, \omega_{y}, \omega_{z}\right]^{T}$, represent the angular velocity of the roll, pitch, yaw paths, $\boldsymbol{\Omega}$ is the representation of absolute angular velocity in body coordinate, and $\gamma=[\varphi, \theta, \psi]^{T}$ is guidance angle of the roll, pitch, yaw, $\boldsymbol{\delta}$ is the deflection angle of the rudder surface, and $\boldsymbol{M}=\left[M_{x}, M_{y}, M_{z}\right]^{T}$ is the control torque.

When $\alpha, \beta$ is known, $\varphi, \theta, \psi$ in attitude coordinate can be converted to $\gamma, \xi, \sigma$ at velocity coordinate, by the following formula (7).

$$
\begin{gathered}
\varphi^{\prime}=\arcsin \left(\cos \gamma \sin \frac{\sigma}{\cos \theta}\right) \\
\phi^{\prime}=\arctan \left(\frac{\sin \sigma \sin \alpha}{\cos \gamma \cos \alpha-\sin \gamma \cos \sigma \sin \alpha}\right)+\xi \\
\theta^{\prime}=\arcsin (\sin \gamma \cos \alpha+\cos \gamma \cos \sigma \sin \alpha) \\
\theta=\arcsin \left(\sin \theta^{\prime} \cos \beta-\cos \theta^{\prime} \cos \varphi^{\prime} \sin \beta\right) \\
\phi=\arctan \left(\frac{\cos \varphi^{\prime} \sin \beta}{\cos \theta^{\prime} \cos \beta+\sin \theta^{\prime} \cos \varphi^{\prime} \sin \beta}\right)+\phi^{\prime} \\
\varphi=\arccos \left(\frac{\cos \theta^{\prime} \cos \varphi^{\prime}}{\cos \theta}\right)
\end{gathered}
$$

\section{B. Object model}

A typical suborbital vehicle as shown in Fig.2, which control rudder surface include elevators, ailerons, yaw rudders, and flap-type plate. Each of these rudder surfaces provide main control torque on the design channel, and have extra influence on other channels. In addition, 38 reaction control system (RCS) injectors designed for auxiliary pitch channel control. Therefore, the object of this paper is extremely complex, and the rudder control allocation with RCS will be the focus of the control law research. 

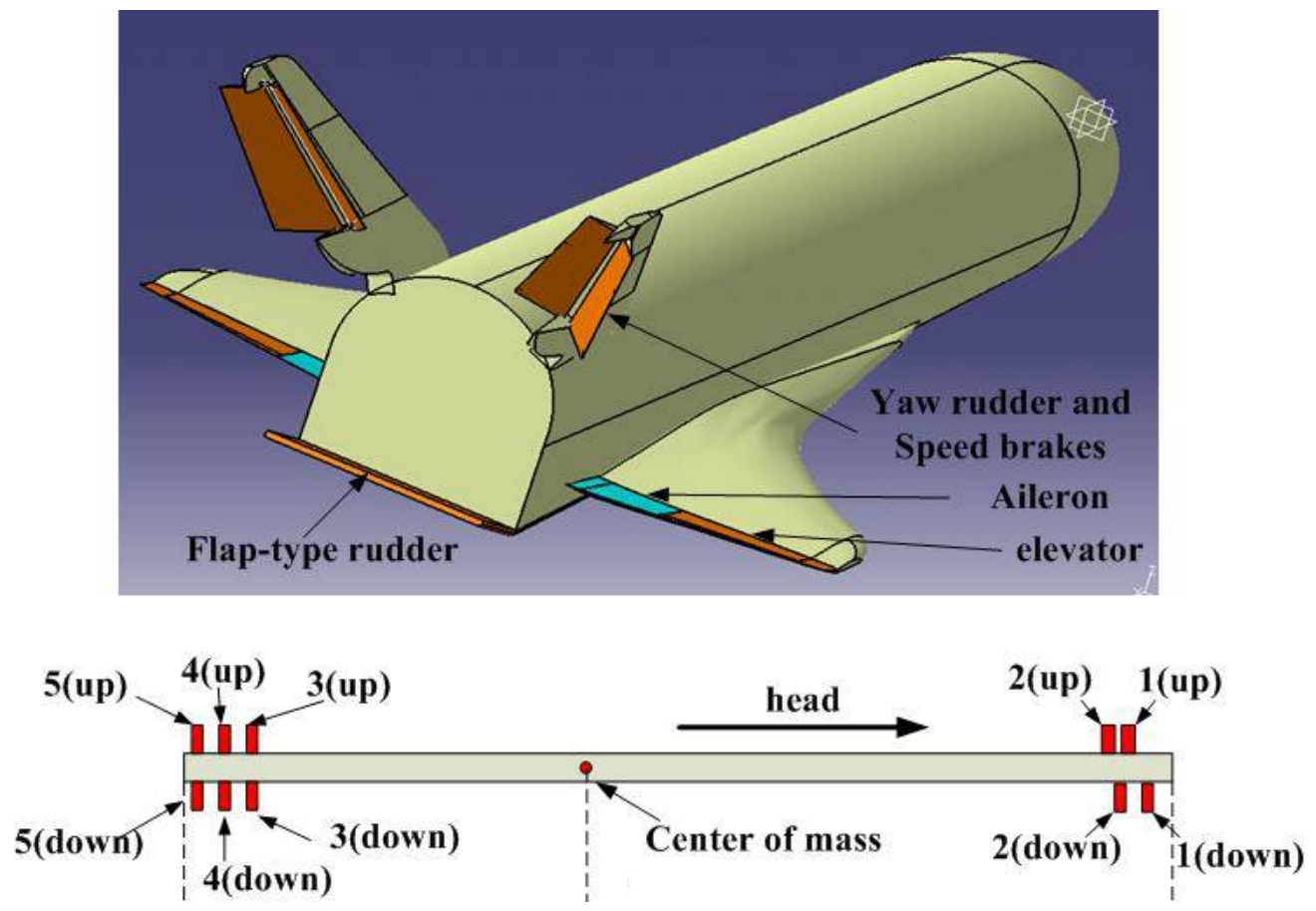

Figure 2. Aerodynamic configuration and control surface of the sub-orbit vehicle (top) and it's RCS injectors (pitch channel) distribution in this paper (bottom).

For this complexity model, it's control torque provided by the rudder surfaces and the RCS, also contains the deflection of $\alpha$ and $\beta$.

$$
\begin{aligned}
& M_{x}=-\frac{1}{2} \rho v^{2} \operatorname{SlCmx} \\
&=-\frac{1}{2} \rho v^{2} S l\left(C m x_{\delta_{-} y a w}+C m x_{\delta_{-} \text {flap }}+C m x_{\delta_{-} \text {elevator }}+C m x_{\delta_{-} \text {aileron }}+C m x_{\alpha, \beta}\right) \\
& M_{y}=-\frac{1}{2} \rho v^{2} S l C m y \\
&=-\frac{1}{2} \rho v^{2} S l\left(\left(C m y_{\delta_{-} \text {yaw }}+C m y_{\delta_{-} \text {flap }}+C m y_{\delta_{-} \text {elevator }}+C m y_{\delta_{-} \text {aileron }}+C m y_{\alpha, \beta}\right)\right) \\
& M_{z}=-\frac{1}{2} \rho v^{2} S l C m z+M_{z_{R C S}} \\
&=-\frac{1}{2} \rho v^{2} S l\left(\left(C m z_{\delta_{-} y a w}+C m z_{\delta_{-} \text {flap }}+C m z_{\delta_{-} \text {elevator }}+C m z_{\delta_{-} \text {aileron }}+C m z_{\alpha, \beta}\right)\right)+M_{Z_{R C S}}
\end{aligned}
$$

\section{Normal Overload Dynamic Balancing Guidance law}

In the traditional study of re-entry flight, we usually take the attack angle as a linear function of velocity

$$
\alpha=\left\{\begin{array}{cc}
\alpha_{0} & V_{e} \leq v<V_{1} \\
\alpha_{0}+k_{\alpha} v & V_{2} \leq v<V_{1} \\
\alpha_{\max _{L / D}} & V_{f} \leq v<V_{2}
\end{array}\right.
$$

In Eq. (9), $V_{e}$ is initial re-entry velocity, $V_{1}$ is the flight velocity when AOA start to adjust, $V_{2}$ is the flight velocity when AOA adjustment end, $V_{f}$ is the velocity at the end of the re-entry, $k_{\alpha}$ is rate of $\mathrm{AOA}(\alpha)$ change with re-entry flight velocity, $V_{1}, V_{2}$ and $k_{\alpha}$ are designed parameters, $\alpha_{0}$ and $\alpha_{\max _{L / D}}$ are constant, correspond to the initial re-entry $\mathrm{AOA}$ and AOA for maximum lift-to-drag ratio flight.

Reference to the U.S. Air Force Space Command's "Suborbital Vehicle Mission for the Mixed-Carrier Program (ARES)", we set the initial conditions for the simulation object as $\{h, v, \gamma\}=\left\{148 \mathrm{~km}, 2133.5 \mathrm{~m} / \mathrm{s}, 0^{\circ}\right\}$, the AOA designed as $\alpha_{0}=40^{\circ}$ before the speed reaches the maximum speed(about $2200 \mathrm{~m} / \mathrm{s}$ ), then reduce $\alpha$ at a linear velocity $k_{\alpha}=25 / \Delta v\left({ }^{\circ} \mathrm{s} / \mathrm{m}\right)$ until the speed reaches the specified value, at this time, $\alpha_{\max _{L / D}}=15^{\circ}$. 
Take $k_{\alpha}$ as different values, the re-entry trajectory results is show in Fig.3.

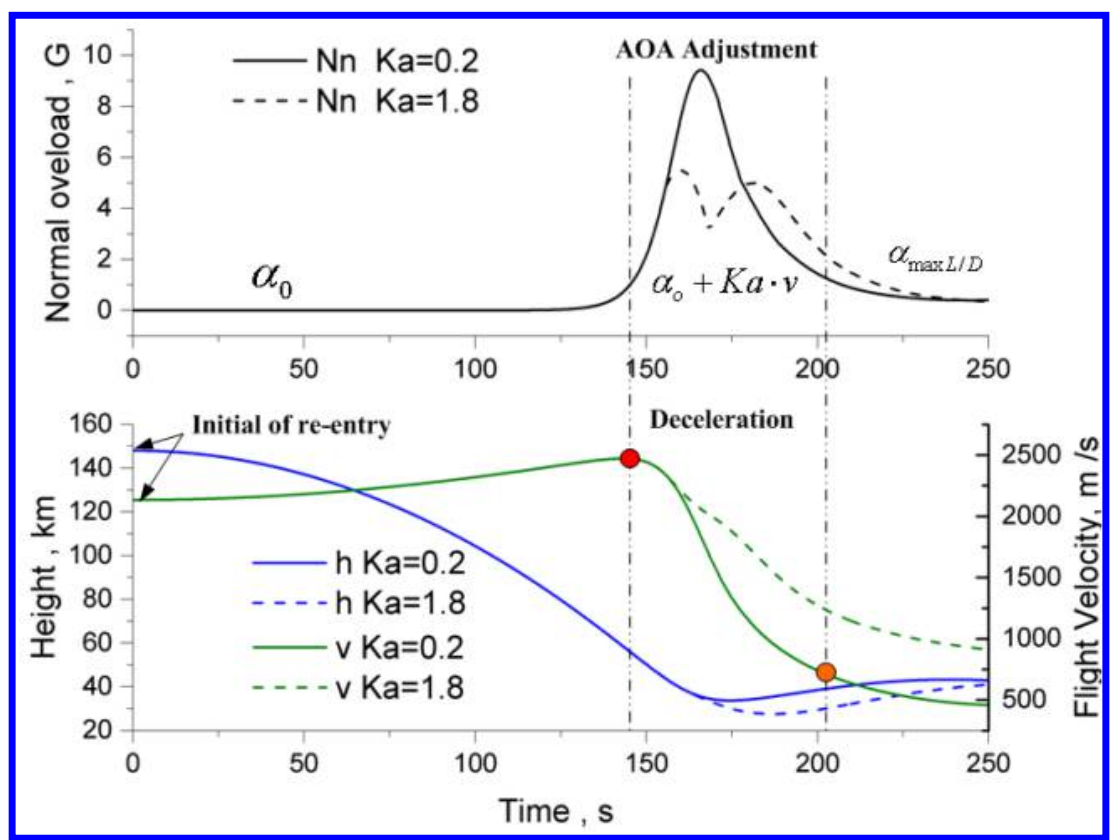

Figure 3. Characteristics of sub-orbital re-entry by using traditional AOA guidance law. Normal overload changes sub-orbital re-entry (top), the re-entry flight height and velocity curve (bottom). AOA adjust with rapid deceleration and normal overload will be a single or double peak value.

It can be seen that when $k_{\alpha}$ changing: the normal overload curves will be a single or double peak value. The reason for this is the decrease of $\alpha$ dose not match with the decrease of velocity. In previous research, we propose a design method of $\alpha$ can make the normal overload fluctuation in the small-scale, its results show that in this condition, the change of $\alpha$ is related both to the velocity and height. Consider that height in the flight model in essence embodied in atmospheric density changes, and the density is positive exponential of height, a formula used to express the initial AOA guidance law.

$$
\alpha(t)=b_{1}+b_{2} v(t)+b_{3} e^{b_{4} h(t)}
$$

In Eq. (10), $\alpha(t)$ is AOA guidance output, $b_{1}, b_{2}, b_{3}$ and $b_{4}$ are fitting parameters determined by designed AOA.

When the normal overload balance value takes different values, $N_{n}=[4.0,4.5,5.0] G$, the angle of attack with the speed and height of the curve shown in Fig.4, and the value of $b_{1}, b_{2}, b_{3}$ and $b_{4}$ are show in Table 1, fitted according to Eq. (10) used least square method. It can be seen theta $\alpha$ is nonlinear but monotonic with velocity and height.

Table 1. The value of $b_{1}, b_{2}, b_{3}$ and $b_{4}$ fitted according to Eq. (10) used least square method, the data used for fitting are the same as Figure 4.

\begin{tabular}{c|cccc}
\hline $\boldsymbol{N}_{\boldsymbol{n}}$ & $\boldsymbol{b}_{\mathbf{1}}$ & $\boldsymbol{b}_{\mathbf{2}}$ & $\boldsymbol{b}_{\mathbf{3}}$ & $\boldsymbol{b}_{\mathbf{4}}$ \\
\hline $\mathbf{4 . 0}$ & 9.54 & $-2.17 \mathrm{E}-03$ & 0.49 & $9.38 \mathrm{E}-05$ \\
$\mathbf{4 . 5}$ & 11.12 & $-3.37 \mathrm{E}-03$ & 0.70 & $8.88 \mathrm{E}-05$ \\
$\mathbf{5 . 0}$ & 13.98 & $-5.57 \mathrm{E}-03$ & 1.05 & $8.27 \mathrm{E}-05$ \\
\hline
\end{tabular}



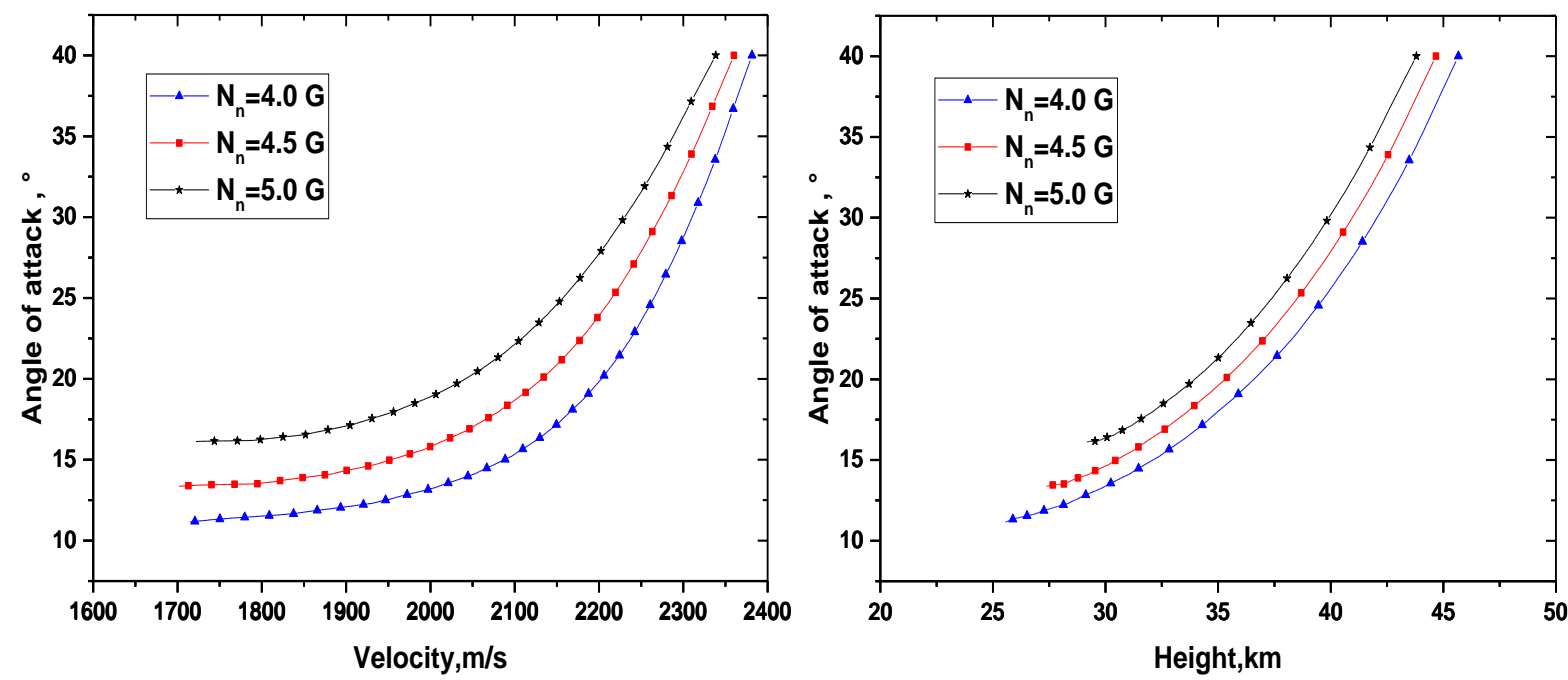

Figure 4. According to the overload balance AOA design method, when the expected equilibrium values are $[4.0,4.5,5.0] \mathrm{G}$, the angle of attack and the velocity, the changing trend between $\alpha$ and $v, \alpha$ and $h$.

Under the condition of no deviation and no disturbance, the fitted formula applied to flight simulation. The normal overload can fluctuate near the predetermined equilibrium value, and the absolute value of the fluctuation error is less than 0.1. However, in the real flight, the uncertainty of the atmospheric data and the aerodynamic coefficients is inevitable. In order to make the guidance law adaptive, we introduce a correction parameters described by the accumulative air resistance during flight. The final guidance law decreased as follows.

$$
\begin{gathered}
\alpha(t)=b_{1}^{\prime}+\left(b_{2} v(t)+b_{3} e^{b_{4} h(t)}\right) \eta(t) \\
\eta(t)=\frac{\int_{t_{0}}^{t} \widehat{D}(t) d t}{\int_{t_{0}}^{t} D(t) d t} \\
b_{1}^{\prime}=\left\{\begin{array}{cc}
a_{\text {start }}-\left(b_{2} v_{\text {satrt }}+b_{3} e^{\left.b_{4} h_{\text {start }}\right) \eta(t)}\right. & t=t_{\text {start }} \\
b_{1}^{\text {start }}+\int_{t_{\text {start }}}^{t} \frac{b_{1} \eta(t)-b_{1}^{\text {start }}}{\Delta T-\left(t-t_{\text {start }}\right)} d t & t_{\text {start }}<t<t_{\text {start }}+\Delta T \\
b_{1} \eta(t) & t \geq t_{\text {start }}+\Delta T
\end{array}\right.
\end{gathered}
$$

$a_{\text {start }}, v_{\text {satrt }}, h_{\text {start }}, t_{\text {start }}$ are corresponding status at start time that $\alpha$ need to adjust in desired trajectory . $\widehat{D}(t)$ is accumulative air resistance in desired trajectory, $\Delta T$ is a presupposed smoothing time, $D(t), v(t)$ and $h(t)$ are actual measurement parameters in re-entry flight.$b_{1}^{\prime}$ is modification of $b_{1}$, that has different value in different stages. 1) Because the initial state is the first point of correction, prone to accumulate large deviations, it needed to fixed initial attack angle at the time when the resistance effect is accumulated to desired value; 2) make smooth change of parameter $b_{1}^{\text {start }}$ to $b_{1} \eta(t)$ after a period of time $\left.\Delta T ; 3\right)$ then maintain $b_{1}^{\prime}=b_{1} \eta(t)$.

Using the same object as earlier, when desired overload $N_{n}=5.0 \mathrm{G}$, the re-entry process using this law is shown in Fig.5.

The simulation result shows that adaptive AOA guidance law is effective, On the one hand, it does not need complex online prediction, so can improve the efficiency of simulation; on the other hand, through the real-time correction of $\eta$ and $b_{1}$, the actual process of aerodynamic deceleration effect is fully taken advantage of. Finally, it still realizing normal overload balance and the fluctuation error is less than 0.1 .

In order to further verify the applicability of this AOA guidance law, for the initial $\{h, v, \gamma\}=$ $\left\{148 \mathrm{~km}, 2133.5 \mathrm{~m} / \mathrm{s}, 0^{\circ}\right\}$, the expected limit of normal overload $4.0,4.5,5.0 \mathrm{G}$ respectively, the overload curve of the re-entry flight process is shown in the Fig.6, there the overload curves are change into platykurtic, and the peak value of the normal acceleration can be remarkably decreased. 


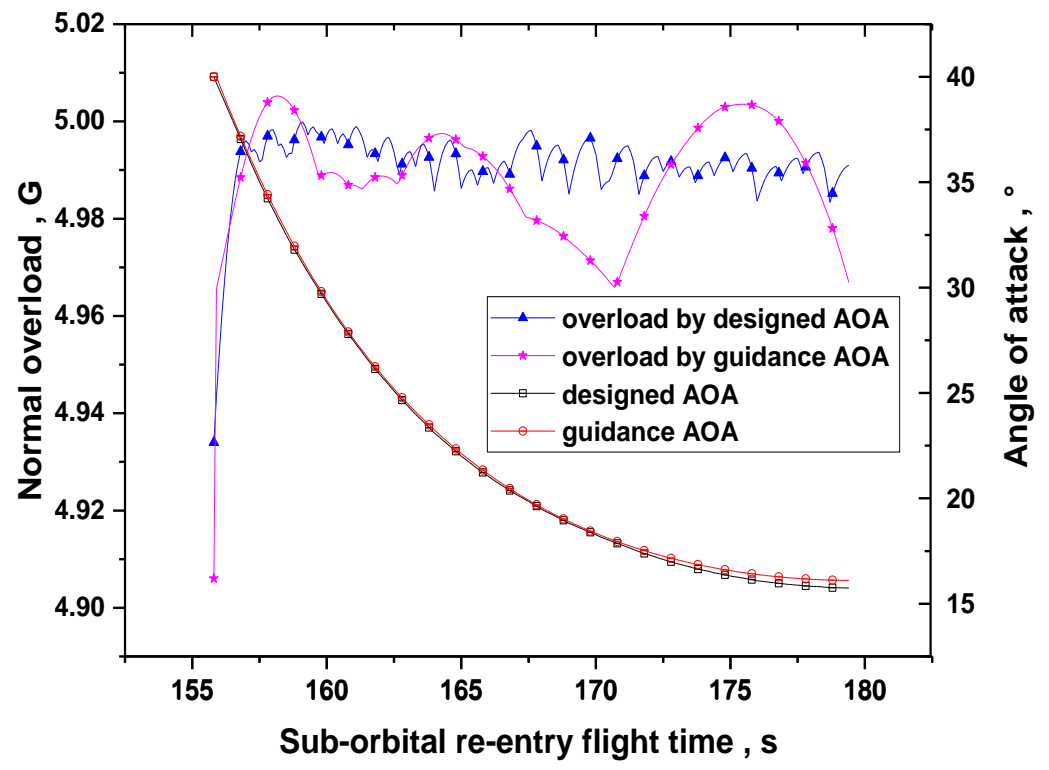

Figure 5. According to the modified AOA guidance law, when $N_{n}=5 \mathrm{G}$, the angle of attack and the normal acceleration in flight are compared with the data before correction.

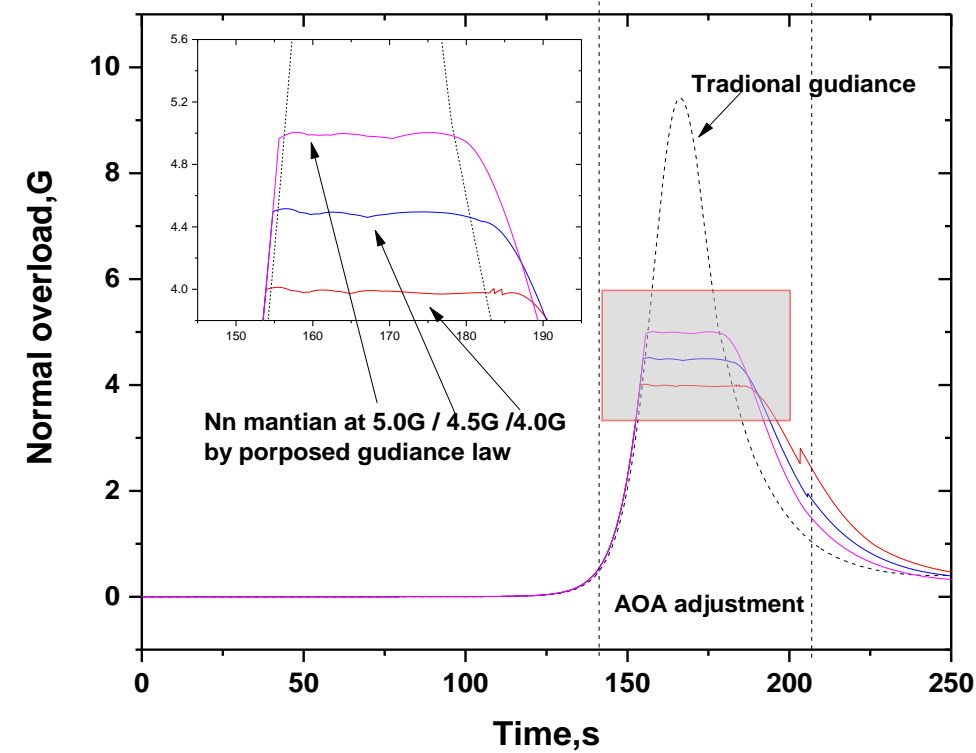

Figure 6. Characteristics of normal overload changes Sub-orbit re-entry by using proposed AOA guidance law, normal overload curves change into platykurtic curve, the peak value of the normal acceleration decreased from 9G to 4G.flight are compared with the data before correction.

\section{Re-entry Controller with multi-rudders and RCS injectors}

Re-entry flight process can be divided into four parts in chronological order: free re-entry, overload control, head alignment phase (HAP) and terminal area energy management (TAEM). During the free re-entry phase $(\mathrm{h}>50 \mathrm{~km})$, aerodynamic force has small influence to re-entry trajectory because of the thin atmospheric density, control objectives is to stable flight attitude; then the sub-orbital vehicle will rapidly entering dense atmosphere without sufficiently decelerate, and the normal overload will rise rapidly, so the primary object in this phase is following normal acceleration reduce guidance law to control overload; as soon as the overload problem solved, the sub-orbital vehicle 
need to adjust the flight heading, the earlier to start HAP, the more re-entry energy will be saved to glide to expected landing area; at the end of the re-entry, sub-orbital vehicle will use terminal area energy management (TAEM) strategy until the landing. A re-entry controller is designed as Fig.7, it contains PIDA controller, double Inverse controller and executing mechanisms distributor.

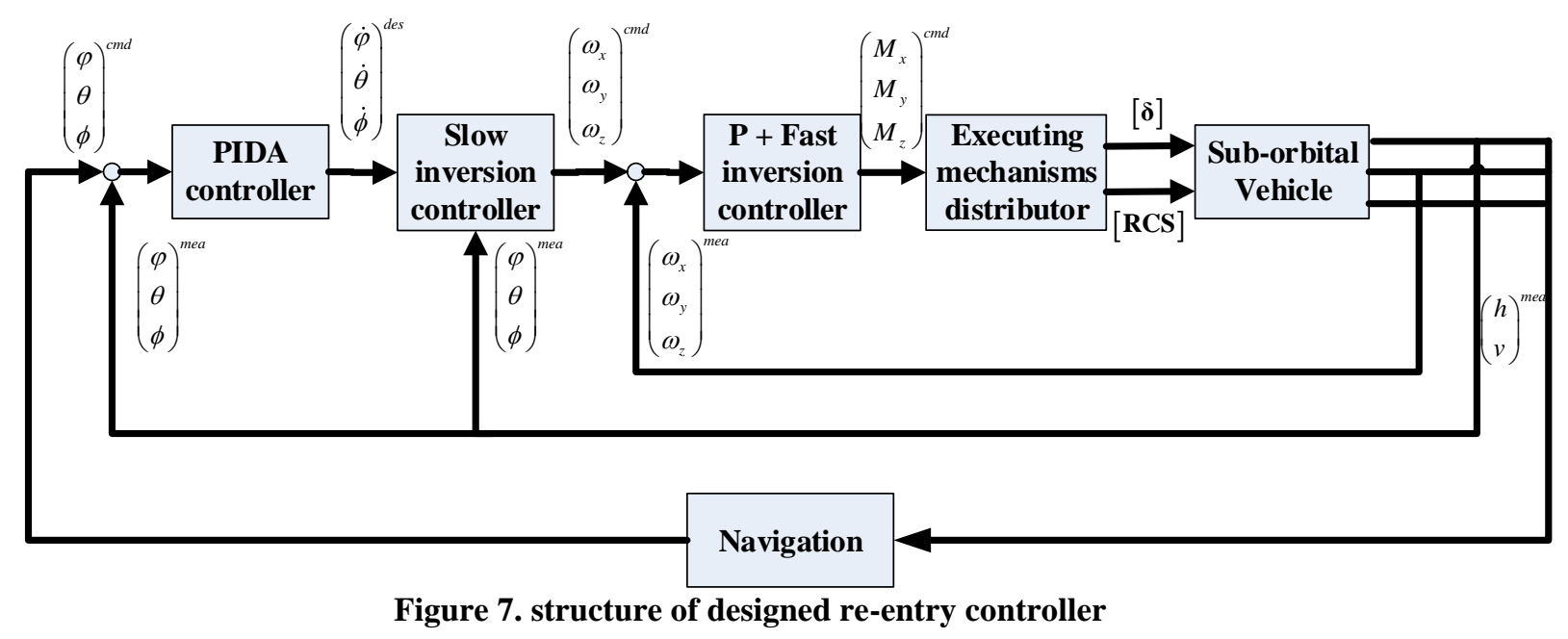

PIDA controller is formula (11):

$$
\begin{gathered}
{\left[\begin{array}{c}
\dot{\varphi} \\
\dot{\theta} \\
\dot{\phi}
\end{array}\right]^{\text {des }}=\left[\begin{array}{c}
k_{P_{-} \varphi} \\
k_{P_{-} \theta} \\
k_{P_{-} \phi}
\end{array}\right] \text { error }_{\varphi, \theta, \phi}+\left[\begin{array}{l}
k_{I_{-} \varphi} \\
k_{I_{-} \theta} \\
k_{I_{-} \phi}
\end{array}\right] \int_{0}^{t} \text { error }_{\varphi, \theta, \phi} d t+\left[\begin{array}{l}
k_{D_{-} \varphi} \\
k_{D_{-} \theta} \\
k_{D_{-} \phi}
\end{array}\right] \frac{\operatorname{derror}_{\varphi, \theta, \phi}}{d t}+\left[\begin{array}{l}
k_{A_{-} \varphi} \\
k_{A_{-} \theta} \\
k_{A_{-} \phi}
\end{array}\right] \frac{d^{2} \operatorname{error}_{\varphi, \theta, \phi}}{d t^{2}}} \\
\operatorname{error}_{\varphi, \theta, \phi}=\left[\begin{array}{c}
\varphi \\
\theta \\
\phi
\end{array}\right]^{\text {cmd }}-\left[\begin{array}{c}
\varphi \\
\theta \\
\phi
\end{array}\right]
\end{gathered}
$$

Slow inversion controller is formula (13):

$$
\begin{gathered}
\omega_{x}^{d e s}=\dot{\varphi}^{\text {des }}+\sin \theta^{\text {mea }} \dot{\phi}^{\text {des }} \\
\omega_{y}^{\text {des }}=\sin \varphi^{\text {mea }} \dot{\theta}^{\text {des }}+\cos \varphi^{\text {mea }} \cos \theta^{\text {mea }} \dot{\phi}^{\text {des }} \\
\omega_{z}^{\text {des }}=\cos \varphi^{\text {mea }} \dot{\theta}^{\text {des }}-\sin \varphi^{\text {mea }} \cos \theta^{\text {mea }} \dot{\phi}^{\text {des }}
\end{gathered}
$$

"P" + fast inversion controller is formula (14):

$$
\begin{gathered}
{\left[\begin{array}{c}
M_{x} \\
M_{y} \\
M_{z}
\end{array}\right]^{\text {des }}=\left[\begin{array}{ccc}
I_{x} & -I_{x y} & -I_{z x} \\
-I_{x y} & I_{y} & -I_{y z} \\
-I_{z x} & -I_{y z} & I_{z}
\end{array}\right]\left[\begin{array}{c}
\dot{\omega}_{x} \\
\dot{\omega}_{y} \\
\dot{\omega}_{z}
\end{array}\right]^{\text {des }}+\left[\begin{array}{c}
\omega_{x} \\
\omega_{y} \\
\omega_{z}
\end{array}\right]^{\text {mea }} \times\left[\begin{array}{ccc}
I_{x} & -I_{x y} & -I_{z x} \\
-I_{x y} & I_{y} & -I_{y z} \\
-I_{z x} & -I_{y z} & I_{z}
\end{array}\right]\left[\begin{array}{c}
\omega_{x} \\
\omega_{y} \\
\omega_{z}
\end{array}\right]^{\text {mea }}} \\
{\left[\begin{array}{c}
\dot{\omega}_{x} \\
\dot{\omega}_{y} \\
\dot{\omega}_{z}
\end{array}\right]^{\text {des }}=\left[\begin{array}{c}
k_{P_{-} \varphi} \\
k_{P_{-}} \\
k_{P_{-}}
\end{array}\right]\left[\left[\begin{array}{c}
\omega_{x} \\
\omega_{y} \\
\omega_{z}
\end{array}\right]^{\text {des }}-\left[\begin{array}{l}
\omega_{x} \\
\omega_{y} \\
\omega_{z}
\end{array}\right]^{\text {mea }}\right.} \\
\omega_{x}^{\text {mea }}=\dot{\varphi}^{\text {mea }}+\sin \theta^{\text {mea }} \dot{\phi}^{\text {mea }} \\
\omega_{y}^{\text {mea }}=\sin \varphi^{\text {mea }} \dot{\theta}^{\text {mea }}+\cos \varphi^{\text {mea }} \cos \theta^{\text {mea }} \dot{\phi}^{\text {mea }} \\
\omega_{z}^{\text {mea }}=\cos \varphi^{\text {mea }} \dot{\theta}^{\text {mea }}-\sin \varphi^{\text {mea }} \cos \theta^{\text {mea }} \dot{\phi}^{\text {mea }}
\end{gathered}
$$

Executing mechanisms distributor is formula (15):

$$
\begin{aligned}
& \delta_{\text {yaw }}^{\text {des }}=\left(-M_{y_{\_} \alpha, \beta}^{\text {mea }}\right) / C m y_{\delta_{-} y a w} \\
& \delta_{\text {flap }}^{\text {des }}=\left\{\begin{array}{cc}
\left(M_{z}^{\text {des }}-M_{z_{-} \alpha, \beta}^{\text {mea }}-M_{z_{-} \delta_{\text {yau }}^{\text {des }}}^{\text {des }}\right) /\left(\frac{1}{2} \rho v^{2} S l C m z_{\delta_{-} \text {flap }}\right) \\
\left|\delta_{\text {flap }}^{\text {max }}\right| \operatorname{sign}\left(M_{z}^{\text {des }}-M_{z_{\alpha}, \beta}^{\text {mea }}-M_{z_{-} \delta_{\text {yaw }}^{\text {des }}}^{\text {des }}\right)
\end{array} \quad\left|\delta_{\text {flap }}^{\text {des }}\right| \leq\left|\delta_{\text {flap }}^{\text {max }}\right|\right.
\end{aligned}
$$




$$
\begin{aligned}
& \delta_{\text {elevator }}^{\text {des }}=\left\{\begin{array}{c}
\left(M_{Z}^{\text {des }}-M_{z_{-} \alpha, \beta}^{\text {mea }}-M_{z_{-} \delta_{\text {yaw }}^{\text {des }}}^{\text {des }}-M_{z_{-} \delta_{\text {flap }}^{\text {des }}}^{\text {des }}\right) /\left(\frac{1}{2} \rho v^{2} S l C m z_{\delta_{-} \text {elevator }}\right) \\
\left|\delta_{\text {elevator }}^{\text {max }}\right| \operatorname{sig} n\left(M_{z}^{\text {des }}-M_{z_{\alpha}, \beta}^{\text {mea }}-M_{z_{-} \delta_{\text {yaw }}^{\text {des }}}^{\text {des }}-M_{z_{-} \delta_{\text {flap }}^{\text {des }}}^{\text {des }}\right)
\end{array}\left|\delta_{\text {elevator }}^{\text {des }}\right| \leq\left|\delta_{\text {elevator }}^{\text {max }}\right|\right. \\
& \delta_{\text {aileron } \pm}^{\text {des }}=\left(M_{x}^{\text {des }}-M_{x_{-} \alpha, \beta}^{\text {mea }}-M_{x_{-} \delta_{\text {yaw }}^{\text {des }}}^{\text {des }}-M_{x_{-} \delta_{\text {flap }}^{\text {des }}}^{\text {des }}-M_{x_{-} \delta_{\text {elevator }}^{\text {des }}}^{\text {des }}\right) /\left(\frac{1}{2} \rho v^{2} S l C m x_{\delta_{-} \text {aileron }}\right) \\
& \delta_{\text {aileron }}^{\text {des }}=\left(M_{z}^{\text {des }}-M_{z_{-} \alpha, \beta}^{\text {mea }}-M_{z_{-} \delta_{\text {yaw }}^{\text {des }}}^{\text {des }}-M_{z_{-} \delta_{\text {flap }}^{\text {des }}}^{\text {des }}-M_{Z_{-} \delta_{\text {elevator }}^{\text {des }}}^{\text {des }}-M_{z_{-} R C S}^{\text {des }}\right) /\left(\frac{1}{2} \rho v^{2} S l C m Z_{\delta_{-} \text {aileron }}\right) \\
& M_{z_{-} R C S}^{\text {des }}=\left.\left(M_{Z}^{\text {cmd }}-M_{z_{\alpha}, \beta}^{\text {mea }}-M_{z_{-} \delta_{\text {yaw }}^{\text {des }}}^{\text {des }}-M_{z_{-} \delta_{\text {flap }}^{\text {des }}}^{\text {des }}-M_{z_{-} \delta_{\text {elevator }}^{\text {des }}}^{\text {des }}\right)\right|_{\text {min }} ^{\text {satisfy }}
\end{aligned}
$$

In Eq. (12) to (16), superscript "cmd" is control input from guidance law, "mea" is control input from navigation measurement, "des" is output of each control loop. In Eq. (12), ( $\left.k_{P_{-} \varphi}, k_{I_{-} \varphi}, k_{D_{-} \varphi}, k_{A_{-} \varphi}\right),\left(k_{P_{-} \theta}, k_{I_{-} \theta}, k_{D_{-} \theta}, k_{D_{-} \theta}\right)$ and ( $\left.k_{P_{-} \phi}, k_{I_{-} \phi}, k_{D_{-} \phi}, k_{D_{-} \phi}\right)$ are PIDA control parameters correspond to roll, pitch and yaw channel. In Eq. (14), $I_{x}, I_{y}, I_{z}, I_{x y}, I_{x z}$ and $I_{y z}$ are moment of inertia of the vehicle, $k_{P_{-} \omega_{x}}, k_{P_{-} \omega_{y}}$ and $k_{P_{-} \omega_{z}}$ are $\mathrm{P}$ parameters correspond to each channel. In Eq. (15), $\delta_{\text {yaw }}^{\text {des }}, \delta_{\text {flap }}^{\text {des }}, \delta_{\text {elevator }}^{\text {des }}, \delta_{\text {aileron } \pm}^{\text {des }}, \delta_{\text {aileron }}^{\text {cmd }}$ and $M_{z_{-} R C S}^{\text {des }}$ are controller output correspond to the yaw rudder angle, flap-type rudder angle, elevator angle, aileron differential angle, aileron angle and RCS injectors. $\left|\delta_{\text {flap }}^{\max }\right|$ and $\left|\delta_{\text {elevator }}^{\max }\right|$ are maxim limit angle to flap-type rudder and elevator, $M_{x_{-} \alpha, \beta}^{\text {mea }}, M_{y_{-} \alpha, \beta}^{\text {mea }}$ and $M_{Z_{-} \alpha, \beta}^{\text {mea }}$ are aerodynamic torques caused by attack and sideslip angle (which can be measured from navigation system and calculate by Eq. (7), similarly $M_{x_{-} \delta_{\text {yaw }}^{\text {des }}}^{\text {des }}, M_{x_{-} \delta_{\text {flap }}^{\text {des }}}^{\text {des }}, M_{x_{-} \delta_{\text {elevator }}^{\text {des }}}^{\text {des }}, M_{z_{-} \delta_{\text {yaw }}^{\text {des }}}^{\text {des }}, M_{z_{-} \delta_{\text {flap }}^{\text {des }}}^{\text {des }}$ are aerodynamic torques caused by yaw rudder, flap-type rudder and elevator in $\mathrm{x}$ and $\mathrm{z}$ channel (body coordinate). $M_{z_{-} R C S}^{\text {des }}$ is obtained from RCS torque table (show as Table 2), $M_{z_{-} R C S}^{\text {des }}$ is the minimum value in Table 2 which satisfies Eq. (16), and the corresponding injects(as show in Fig. 2) in Table 2 will be used.

\begin{tabular}{|c|c|c|c|}
\hline Serial number & RCS injectors simultaneous action & Torque direction & Torque value (Nm) \\
\hline 1 & 5up+4down & Upward & 800 \\
\hline 2 & 5 up +3 down & Upward & 1600 \\
\hline 3 & 5 up+1up & Upward & 3290 \\
\hline 4 & 5 down +2 down & Upward & 17677 \\
\hline 5 & 3up & Upward & 30332 \\
\hline 6 & 3up+4up & Upward & 61463 \\
\hline 7 & $3 u p+4 u p+5 u p$ & Upward & 93395 \\
\hline 8 & 2 down $+3 u p+4 u p+5 u p$ & Upward & 143003 \\
\hline 9 & 1 down +2 down +3 up +4 up +5 up & Upward & 193651 \\
\hline 10 & 4 up +5 down & Downward & 800 \\
\hline 11 & 3 up +5 down & Downward & 1600 \\
\hline 12 & 2 up +2 down & Downward & 7674 \\
\hline 13 & 2 down+4down+5down & Downward & 13455 \\
\hline 14 & 3down & Downward & 30332 \\
\hline 15 & 3 down +4 down & Downward & 61463 \\
\hline 16 & 3 down +4 down +5 down & Downward & 93395 \\
\hline 17 & 1up +3 down +4 down +5 down & Downward & 122046 \\
\hline 18 & 1 up $+2 u p+3$ down +4 down +5 down & Downward & 179329 \\
\hline
\end{tabular}

$$
\left|M_{z_{-} R C S}^{\text {des }}+M_{z_{-} \delta_{\text {flap }}^{\text {des }}}^{\text {des }}+M_{Z_{-} \delta_{\text {elevator }}^{\text {des }}}^{\text {des }}\right| \geq\left|M_{z}^{\text {des }}\right|
$$

Table 2. RCS injectors' simultaneous action combinations table 


\section{Sub-orbital re-entry flight 6 DOF simulation results analysis}

The 6 DOF simulation use the U.S standard atmosphere 1976 model and the HWM07 wind field model, the RCS injectors parameters are shown in Table 3, the rudders transfer function are considered as first-order process with time delay (characteristic time constant is 0.05). Suborbital vehicle re-entry initial status and required ending status are shown in Table 3, rudders angle limits are shown in Table 4. The normal overload setting value during overload control phase is $5 \mathrm{G}$.

Simulation example 1: $6 \mathrm{DOF}$ re-entry simulation use the nominal aerodynamic elements and without use wind model.
Table 3. Parameters of RCS injectors in 6 DOF simulations

\begin{tabular}{c|cc}
\hline Parameters & Unit & Value \\
\hline $\begin{array}{c}\text { Thrust deviation range } \\
\text { Maximum Time for continuously } \\
\text { working }\end{array}$ & s & 10 \\
Restart time delay & & 120 \\
Minimum work time & s & 0.01 \\
Limit of repeat & s & 0.01 \\
Working synchronicity & $/$ & 5000 \\
ISP (in vacuum) & $\mathrm{ms}$ & $\leqslant 2$ \\
Response time & $\mathrm{s}$ & 280 \\
\hline
\end{tabular}

Table 4. Sub-orbital vehicle re-entry initial status and requirements in 6 DOF simulations

\begin{tabular}{c|cc}
\hline & Reentry initial status & required ending status \\
\hline Height & $148 \pm 2 \mathrm{~km}$ & $3 \pm 0.02 \mathrm{~km}$ \\
Velocity & $2133 \pm 20 \mathrm{~m} / \mathrm{s}$ & $150 \pm 5 \mathrm{~m} / \mathrm{s}$ \\
Path angle & $0 \pm 0.1^{\circ}$ & $>-25^{\circ}$ \\
Course angle (from north) & $192.9^{\circ}$ & $<$ \\
Longitude and latitude (initial) \\
Distance to land area (ending)
\end{tabular}

The results (flight height, velocity normal overload and attitude angle control results are shown in Fig. 8 , multiple rudders and RCS injectors control status are shown in Fig.9. The simulation result indicates effectiveness of the proposed algorithm: the normal overload is near the setting value and fluctuates in small scope by the proposed guidance law , three axis attitude of vehicle are tracking well with guidance command during the entire re-entry flight , peak error of pitch, yaw, roll channel are less than $0.5^{\circ}, 1.0^{\circ}$ and $0.15^{\circ}$, respectively (as shown in Fig.8).

Table 5. Sub-orbital vehicle rudders angle limits in 6 DOF simulations

\begin{tabular}{c|c}
\hline rudders & angle limits \\
\hline yaw rudder & $-20^{\circ}-20^{\circ}$ \\
flap-type rudder & $-20^{\circ}-25^{\circ}$ \\
elevator & $-30^{\circ}-30^{\circ}$ \\
aileron differential angler & $-15^{\circ}-15^{\circ}$ \\
aileron & $-20^{\circ}-20^{\circ}$ \\
Speed brakes & $0^{\circ}-150^{\circ}$ \\
\hline
\end{tabular}

10

American Institute of Aeronautics and Astronautics 


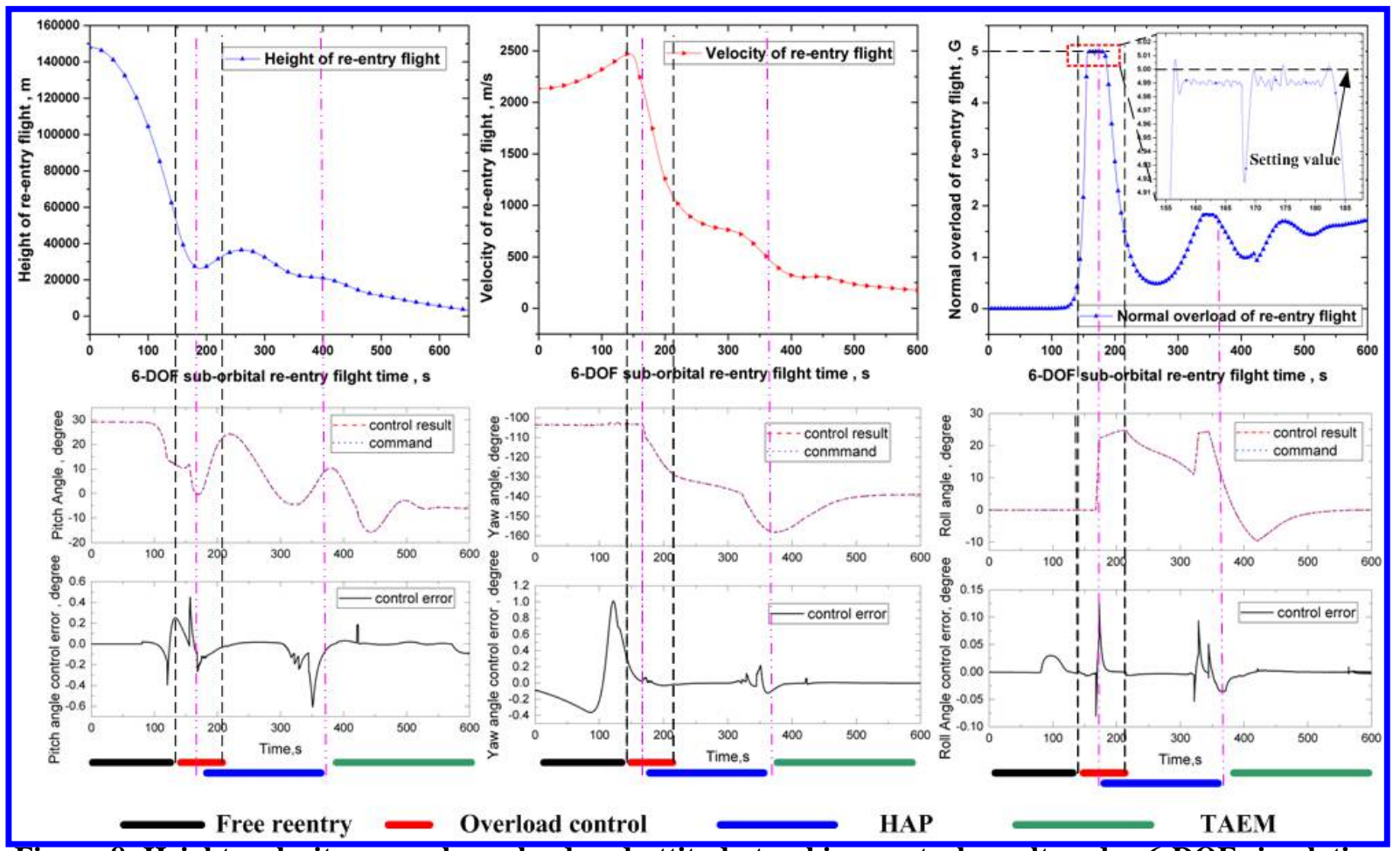

Figure 8. Height, velocity normal overload and attitude tracking control result under 6 DOF simulation during the entire process of sub-orbit vehicle re-entry. The re-entry is divided into several stages: Free reentry, Overload control, Head Alignment Phase (HAP) and Terminal area energy management (TAEM).

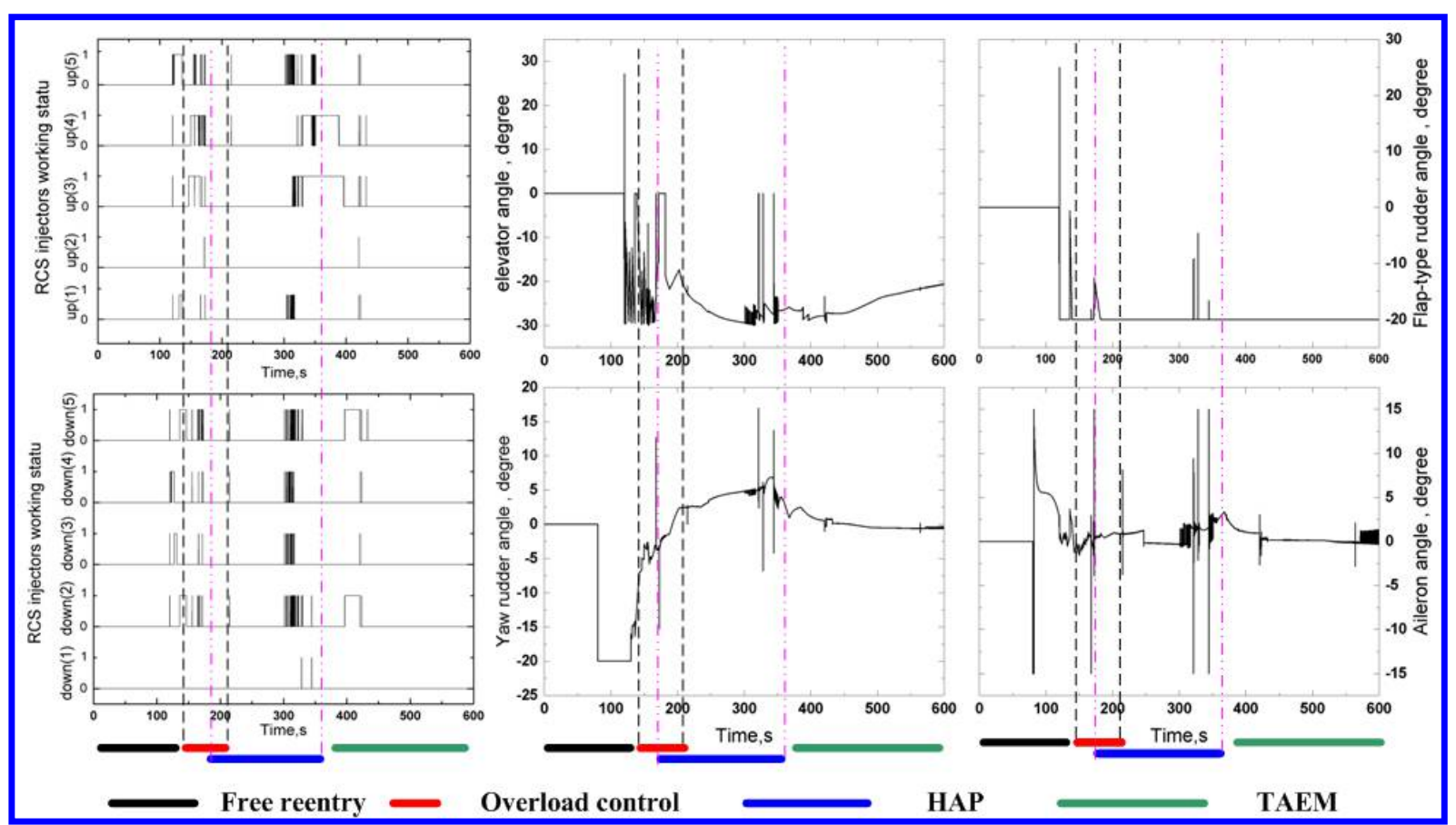

Figure 9. RSC working status (left), Elevator angle (middle top), Yaw rudder angle (middle bottom), Flagtype rudder angle (right top) and Aileron angle (right bottom) control result under 6 DOF simulation during the entire process of sub-orbit vehicle re-entry. 
Simulation example 2: normal overload 3 DOF (guidance only) and 6 DOF (guidance \& control) re-entry simulation (result are shown in Fig. 10 right), and 6 DOF simulation with $\pm 20 \%$ aerodynamic elements uncertainty ( result are shown in Fig.10 left). The result of 3 DOF and 6 DOF comparison show that control will shorten the reentry flight process. Both 3 DOF and 6 DOF normal overload are tracking well to the setting overload value (5G), even in the $\pm 20 \%$ aerodynamic elements uncertainty conditions.
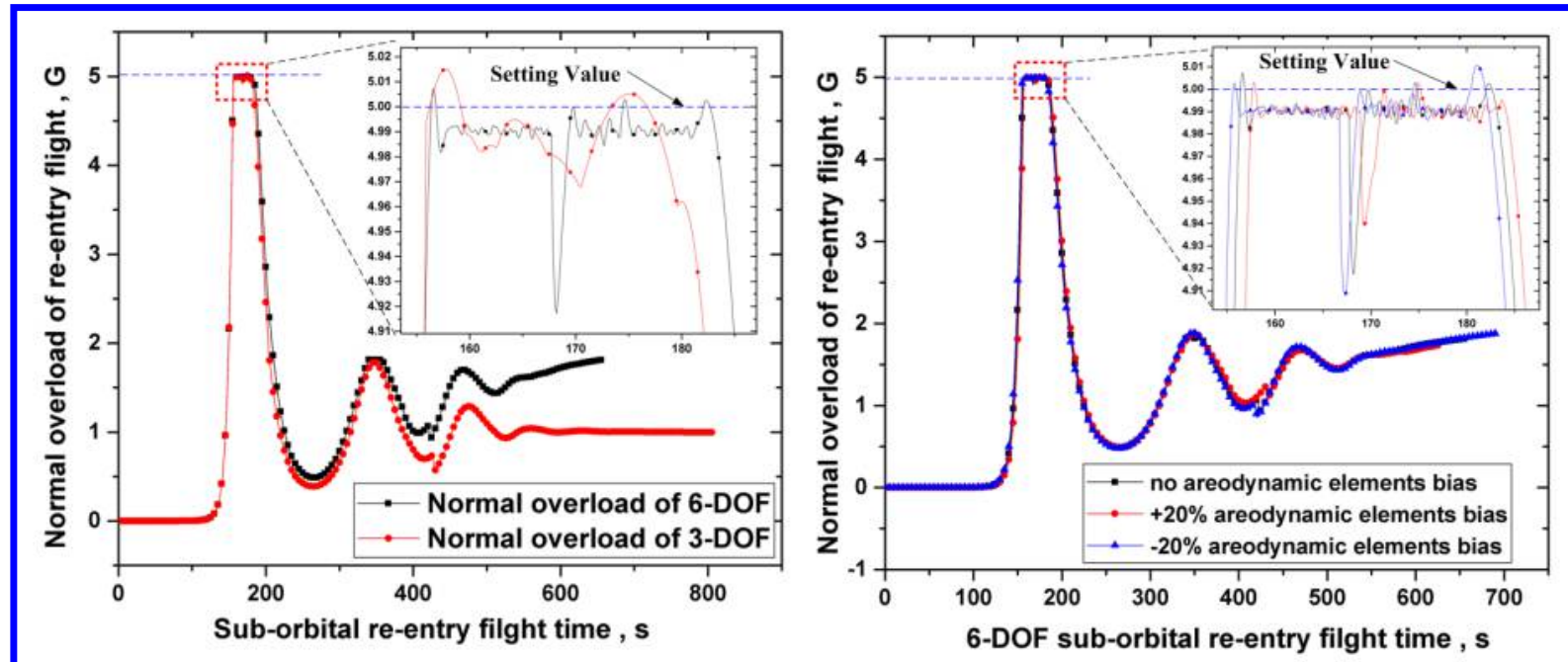

Figure 10. Normal overload results of 3-DOF and $6 \mathrm{DOF}$ compare simulation (left), 6 DOF simulation with $\pm 20 \%$ aerodynamic elements uncertainty (right) during the entire process of sub-orbit vehicle re-entry.

Simulation example 3: Monte-Carlo 6 DOF re-entry simulations with $\pm 20 \%$ aerodynamic elements uncertainty and random wind. The results indicate the effectiveness of the proposed guidance and control method: required ending status are well satisfy and the peak normal overload value have good performance in following setting value (deviation is less than 6\%).
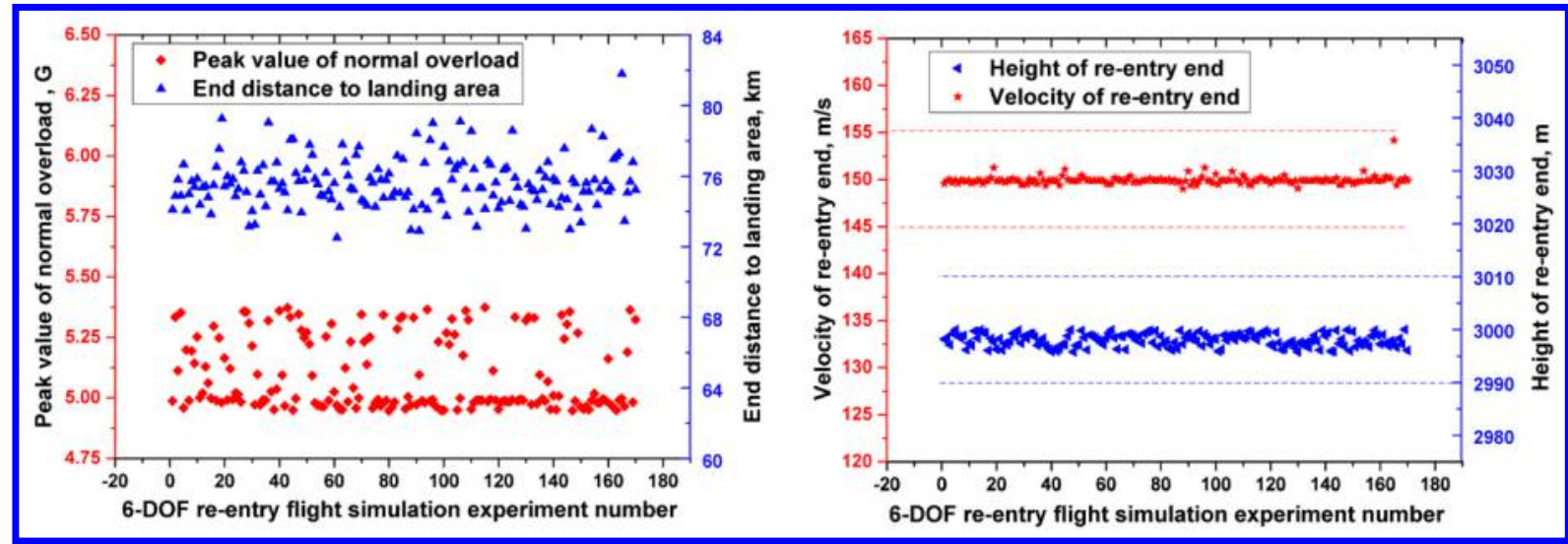

Figure 11. Monte-Carlo 6 DOF re-entry simulations results with $\pm 20 \%$ aerodynamic elements uncertainty and random wind. Peak value of normal overload and ending distance to landing area results (left), ending height and velocity results (right). The dotted lines in right fig is the corresponding limits.

\section{Conclusions and discussions}

In this paper, a new AOA guidance law and controller with multi-control surface and RCS are designed to reduce normal acceleration in sub-orbital re-entry phase. Simulation results shows that the proposed method can effectively control re-entry trajectories and attitude, the setting normal overload value is setting as $5 \mathrm{G}$ in simulations so to satisfy multiple ending status requirements. The normal overload can be setting even lower (as show in Fig.6, can be lower than $4 \mathrm{G}$ ) by changing curves into platykurtic curve, and the proposed guidance and control method may be used to reach the boundary of the region with strict normal overload constrains. 


\section{References}

${ }^{1}$ Seedhouse, E., SpaceShipTwo: VSS Enterprise. Virgin Galactic. Springer International Publishing, 2015:65-85.

${ }^{2}$ Thorpe, D. G., Rhodes, R. E., Robinson, J. W.,"Supersonic Sub-Orbital Aircraft for Earth to Orbit mission." Aiaa/asme/sae/asee Joint Propulsion Conference 2015.

${ }^{3}$ Leavitt J. A., "Advanced entry guidance algorithm with landing footprint computation”. 2005.

${ }^{4} \mathrm{Li}, \mathrm{H}$., and Xie L., "Reentry guidance law design for RLV based on predictor-corrector method." Journal of Beijing University of Aeronautics \& Astronautics 35.11(2009):1344-1348.

${ }^{5}$ Liu Q J, Huang Y M, Liao-Ni W U., "Research on Upper-Limit of Altitude on Suborbital Reentry." Journal of Astronautics (2010).

${ }^{6}$ Xiao, X. X., Wen-Hao, L. I., Zhang, H.,"Study of reentry channel for sub-orbital vehicle." Flight Dynamics 29.3(2011):60-63.

${ }^{7}$ Xiao, X. X., Li, W. H., Zhang, H., "Research on attack angle design method for sub-orbital vehicle re-entry to reduce the normal acceleration peak value." Journal of Astronautics 43.4(2012):345-351.

${ }^{8} \mathrm{Wu}, \mathrm{L}$., Huang, Y., Zhang, J.,"The Design of Guidance on Suborbital Reentry." International Asia Conference on Informatics in Control, Automation and Robotics, Car 2009, Bangkok, Thailand, 1-2 February DBLP, 2009:402-405.

${ }^{9} \mathrm{Li}$, W. H., Zhang, H., "Reentry flight control method based on PIDA policy." Journal of System Simulation 23.5(2011):969972. 